\title{
Androgen Stimulated Chemotherapy in the Dunning R-3327 Prostatic Adenocarcinoma
}

\author{
H. Barton Grossman ${ }^{1, *, * * *}$, Edward L. Kleinert ${ }^{1}$, Martin L. Lesser ${ }^{2}$, Harry W. Herr ${ }^{1}$ \\ and Willet F. Whitmore, Jr. \\ ${ }^{1}$ Section of Urology and ${ }^{2}$ Biostatistics Laboratory, Memorial Sloan-Kettering Cancer Center, \\ New York, New York, USA
}

Accepted: February 24, 1981

Summary. This study was undertaken to determine whether hormonal stimulation followed by chemotherapy with a cell-cycle specific agent would improve the effectiveness of the chemotherapy in a prostatic adenocarcinoma model.

One hundred Copenhagen rats were randomised into 5 equal groups and injected subcutaneously with $2 \times 10^{7}$ cells of Dunning G strain prostatic adenocarcinoma. The groups were treated in the following fashion: 1 . sham operated controls, 2. castration, 3. castration and methotrexate, 4. castration, testosterone and methotrexate and 5 . castration and testosterone. When the tumours became palpable, all animals received the surgery to which they were randomised. Subsequent hormonal and chemotherapy was started 1 week thereafter. Therapy was given for 5 consecutive days followed by a 16 -day recovery period and then continued in a cyclical fashion. Serial measurements of animal weights and tumour size were obtained. Analysis of tumour growth was restricted to the first 29 days of therapy because of a rapid decline in animal survival beyond that point.

The group treated with castration, testosterone, and methotrexate inhibited tumour growth more than any other group and was the only group that was significantly different from control $(\mathrm{P}<0.05)$.
Key words: R-3327 prostatic adenocarcinoma, Chemotherapy, Hormonal stimulation.

\section{INTRODUCTION}

The Dunning $\mathrm{R}-3327$ prostatic carcinoma originated from a spontaneous neoplasm in an aged Copenhagen rat (3). The $G$ sub-line has been characterised as a rapidly-growing transplantable tumour that is hormonally responsive (1). This study was undertaken to evaluate the effects of androgen on the response of the tumour to chemotherapy with a cell cycle-specific agent in the previously castrated animal. The rate of DNA synthesis in prostatic tissue of castrated rats increases $24 \mathrm{~h}$ following daily parenteral exogenous testosterone administration and peaks at $72 \mathrm{~h}(2)$. To assess the effect of androgen on the response of the hormonally responsive prostatic neoplasm to chemotherapy, castrated Copenhagen rats bearing transplanted $G$ strain tumours were treated with testosterone and/or methotrexate with appropriate controls.

\section{MATERIAL AND METHODS}

One hundred Copenhagen rats ${ }^{1}$, age 14 weeks, were randomly assigned to 5 groups of 20 each and injected subcutaneously in the left flank with $2 \times 107$ cells of R-3327-G prostatic adenocarcinoma ${ }^{2}$. The cell suspension was prepared by

\footnotetext{
*Dr. Grossman is a Ferdinand C. Valentine Fellow of the Section of Urology, New York Academy of Medicine

* Send reprint request to Dr. Grossman at his current address: Section of Urology, University of Michigan Medical Center, 1405 East Ann Street, Box 03, Ann Arbor, Michigan 48109, USA
}

\footnotetext{
The Copenhagen rats were obtained from the National Cancer Institute, Bethesda, Maryland ${ }^{2}$ The R-3327-G cell line was obtained from $\mathrm{Dr}$. Norman Block, Jackson Memorial Hospital, Miami, Florida and has been maintained in our laboratory by serial transplantation in Copenhagen rats since 1977
} 
mincing freshly excised tumour from a stock animal in RPIMI 1640. The minced tumour was dispersed by stirring in $0.5 \%$ trypsin with $0.02 \%$ collagenase at room temperature. The resulting mixture was filtered through gauze to eliminate large pieces of tumour, washed once in RPMI 1640 , and viable cell counts then determined by trypan blue exclusion. The cells were diluted with RPMI 1640 to a concentration of $2 \times 10^{7}$ cells $/ 0.5 \mathrm{ml}$. All animals had palpable evidence of tumours at the injection site 2 weeks thereafter.

When the tumours first became palpable (mean diam. $0.74 \mathrm{~cm}$ ) all animals either underwent a sham operation (Group 1) or were castrated. Groups 1 (sham operated) and 2 (castrated) received no additional therapy. The remainder of the castrated animals received additional hormonal and/or chemotherapy. Groups 3 and 4 received $0.58 \mathrm{mg} / \mathrm{kg}$ methotrexate intraperitoneally for 5 consecutive days followed by a 16 day rest period, this regimen being continued until death. Animals in Group 4 received, in addition, $2 \mathrm{mg}$ testosterone propionate in sesame oil subcutaneously $1 \mathrm{~h}$ prior to each dose of methotrexate. Group 5 received testosterone but no methotrexate. Sham operation or castration was performed on day 1 and chemotherapy and/or hormonal therapy in Groups 3-5 was instituted on day 8 of the experiment. All animals were weighed weekly and had tumours measured twice weekly in three axes by the same observer using a caliper. All animals were followed until death (Fig. 1).

Fifteen animals examined shortly after death had tumour measured in the standard fashion and in addition had an actual measurement of tumour volume by excision of the tumour and volume displacement.

Analysis of tumour growth involved calculation of relative tumour size over time and comparison of the resultant areas under the curve (4). Relative tumour size is the ratio of actual tumour size to that on the day treatment was commenced.

\section{RESULTS}

Analysis of tumour growth was restricted to the first 29 days because of a rapid decline in animal survival beyond this point (Fig. 2). The median survival times for Groups 1-5 respectively were $36,37,39,33$ and 35 days (Fig. 3). Ten animals were excluded from analysis because of death prior to day 29. Of these 10 animals 3 were in Group 1, 3 in Group 3 and 4 in Group 4. No differences in the patterns of tumour growth were seen in these rats when compared to surviving animals in the respective groups, and the cause of death was not determined.

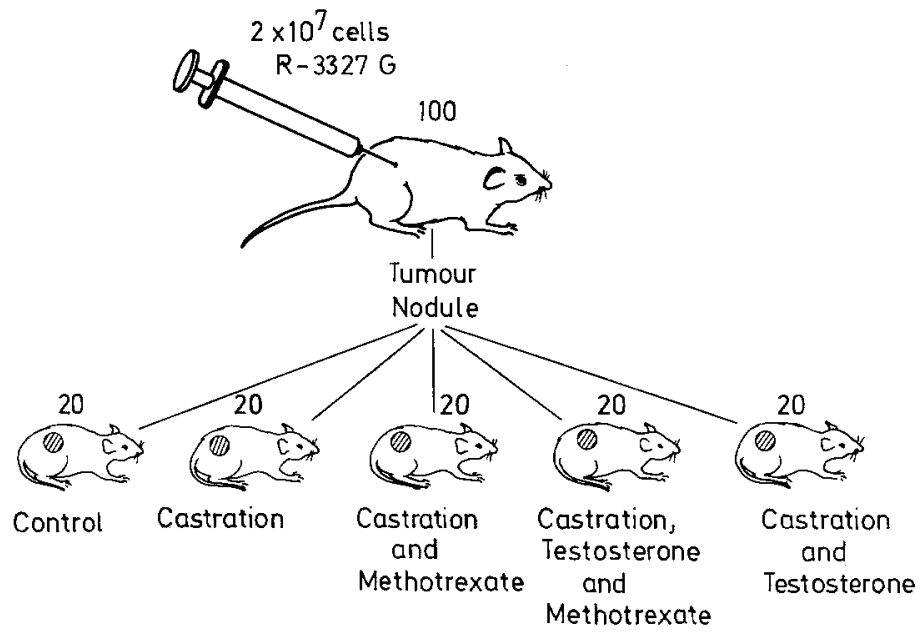

Fig. 1. Illustration of tumour induction and treatment protocol

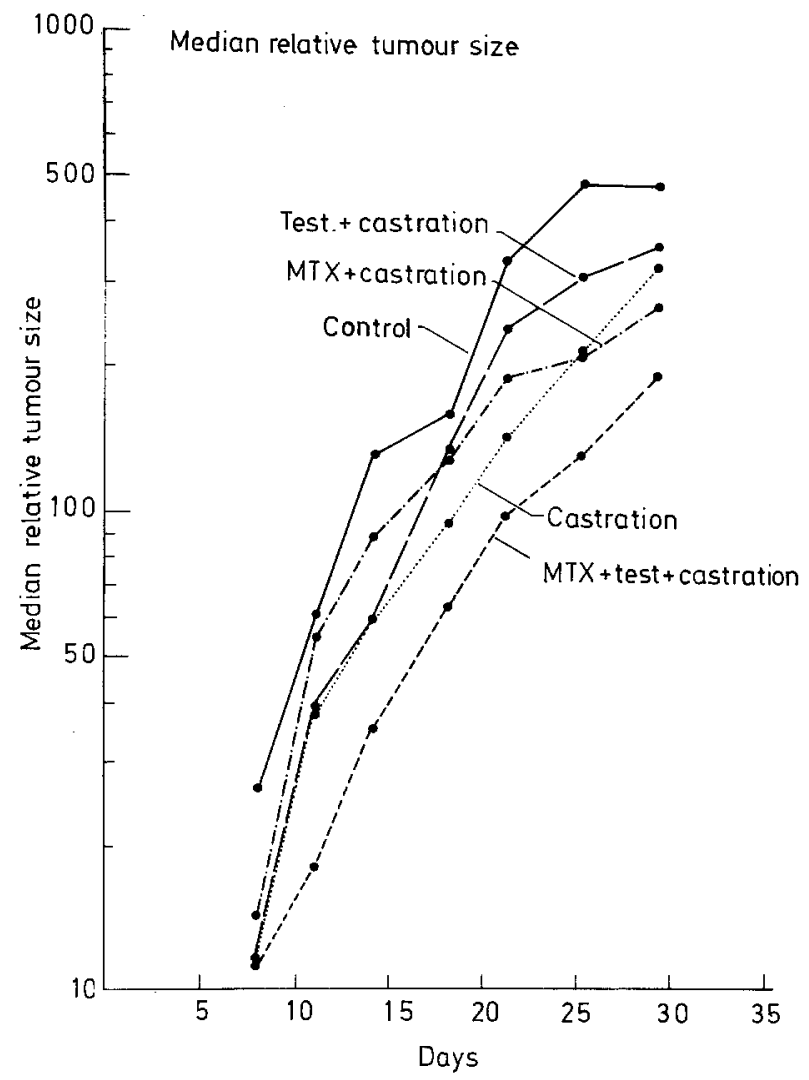

Fig. 2. Median relative tumour size for all treatment groups. Test. $=$ testosterone, MTX $=$ methotrexate

The mean areas under the growth curves for Groups 1-5 respectively, were 7126.43, 4180.05, 4008.93, 2158. 86 and 5380.18. The group receiving castration, testosterone and methotrexate (Group 4) exhibited the least tumour growth and was the only group significantly different from control (Kruskal-Wallis test, $\mathrm{p}<0.04$ and Bon- 
Animal survival

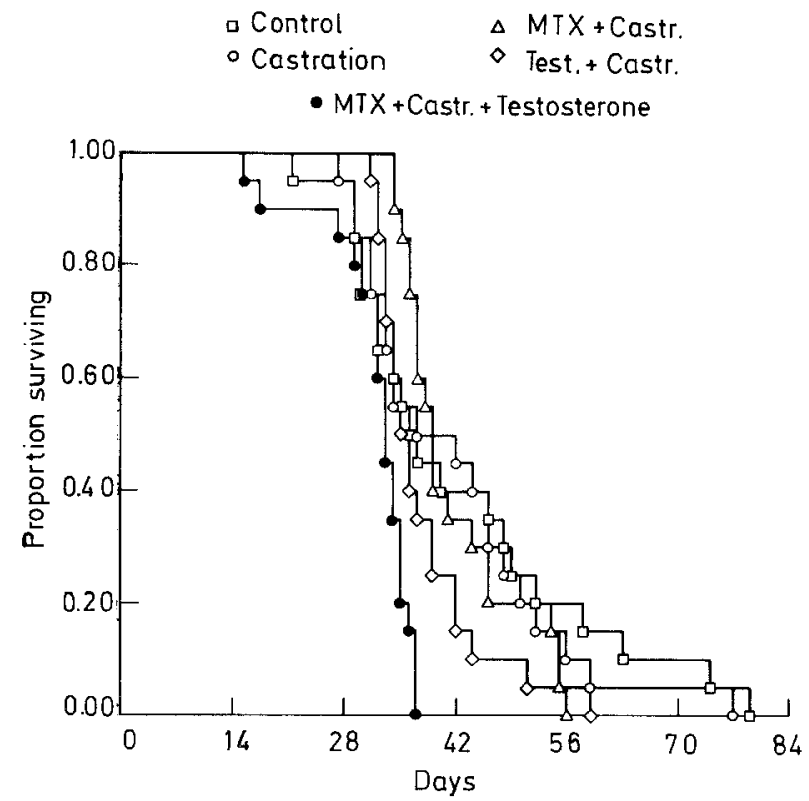

Fig. 3. Animal survival for all treatment groups. Test. = testosterone, MTX = methtrexate, Castr. = castration

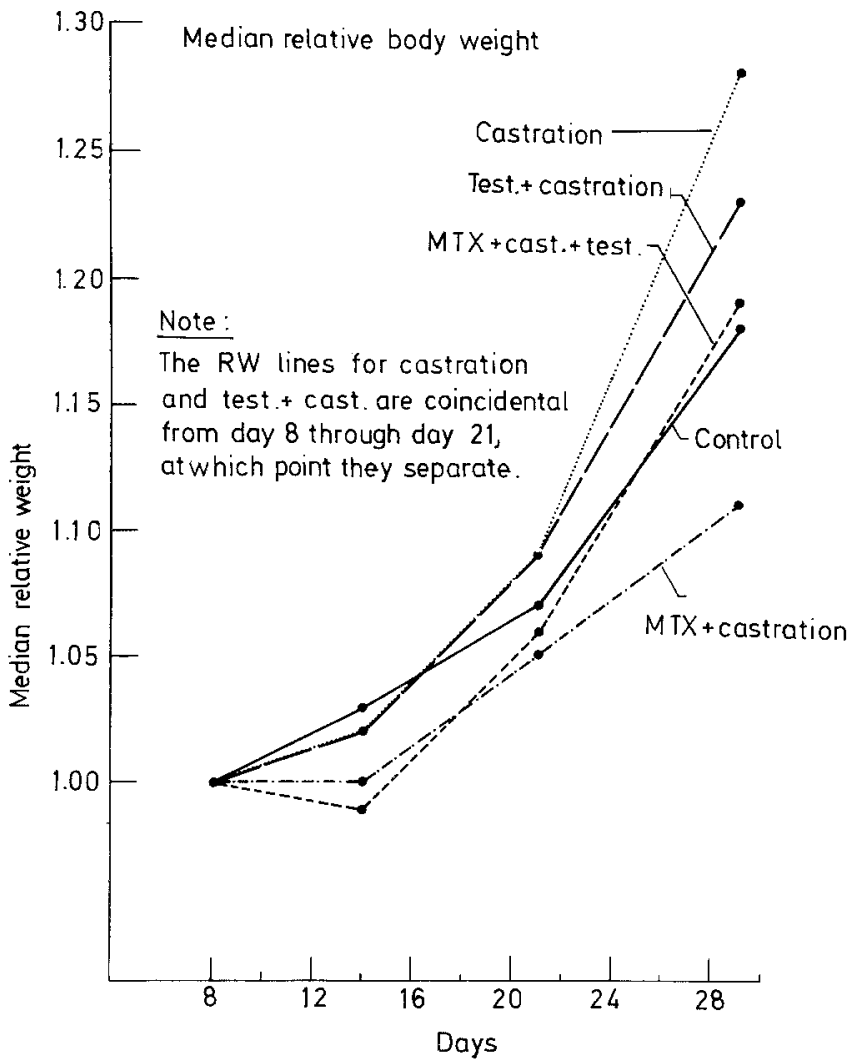

Fig. 4. Median relative body weight for all treatment groups. Test. = testosterone, MTX $=$ methotrexate, Castr. = castration ferroni method of multiple comparisons using the Mann-Whitney test, $\mathrm{p}<0.05)$.

Analysis of animal weights was carried out using the same technique as previously described for tumour growth (Fig. 4) and revealed no significant differences among relative weight curves using the "area under the curve" method.

For the 15 rats used to assess the correlation of in situ measurement of tumour size at time of death with actual volume, the Spearman rank correlation coefficient was $r=0.85$, which indicated a strong positive correlation between the 2 methods $(p<0,01)$.

\section{DISCUSSION}

Analysis of tumour growth curves in the R-3327-G model revealed that the group receiving castration, testosterone and methotrexate (Group 4) had the least tumour growth and was the only group significantly different from control. The use of testosterone apparently increased the therapeutic effectiveness of the subsequent cell cycle specific chemotherapy. Several explanations can be offered for these results:

1. Testosterone decreased tumour growth independently and added to the effectiveness of the subsequent chemotherapy. This possibility is eliminated by the fact that the addition of testosterone to castration increased, if anything, tumour growth more than castration alone.

2. Testosterone acted synergistically with methotrexate to decrease tumour growth. Testosterone is essential for the growth and maintenance of the mammalian prostate and results in increased DNA synthesis in the prostate of castrated rats. The androgen responsive Dunning R-3327-G prostatic adenocarcinoma presumably behaves similarly. The stimulation of DNA synthesis and mobilisation of cells into an actively dividing pool may have increased the effectiveness of methotrexate which is primarily toxic to cells in the $S$ phase of the cell cycle (5). Alternatively, testosterone may have increased the cellular uptake of methotrexate by the tumour in a fashion unrelated to events in the cell cycle or a combination of these events may have occurred.

Animal survival is an event related to tumour growth and chemotherapy but is not entirely dependent on these factors. The failure to document increased survival in Group 4 does not appear to be related to tumour growth because of the salutary effect of the treatment programme in this group. Testosterone and methotrexate in this regimen are not toxic to intact, nontumour bearing animals (unpublished data) but the effect on tumour bearing animals is unknown. Further modifications of the treatment programme and/or the addition of supportive care (careful monitoring of fluid and electrolyte balance, etc.) may allow the more definitive 
separation of these 2 events, i. e. survival and tumour growth, while maintaining the apparent advantage in tumour control.

\section{REFERENCES}

1. Camuzzi, F., Block, N.L., Stover, B., Gottlieb, C., Charyulu, K., Politano, V.A. : Investigation of different combinations of estrogen therapy and radiation therapy on prostatic adenocarcinoma (R-3327). Urology 15,443 (1980)

2. Coffey, P.S., Shimazaki, J., WilliamsAshman, H. G. : Polymerization of deoxribonucleotides in relation to androgen-induced prostatic growth. Archives of Biochemistry and Biophysics 124, 184 (1968)

3. Dunning, W. F. : Prostate cancer in the rat. National Cancer Institute Monographs 12, 351 (1963)
4. Lesser, M. L., Braun, H. I., Helson, L. : Statistical methods for measuring and comparing treatment efficacies: Applications to nude mice experimentation. Experimental Cell Biology 45, 126 (1980)

5. Skipper, H.T., Schabel, F. M., Jr.: Quantitative and cytokinetic studies in experimental tumor models. In: Cancer Medicine. Holland, J. F., Frei, E., III (eds.), p. 629. Philadelphia: Lea and Febiger 1973

Dr. H. B. Grossman

Section of Urology

University of Michigan Medical Center

1405 East Ann Street

Box 03

Ann Arbor, Michigan 48109

USA 